\title{
A Theoretical Study of the Cosmic Expansion in the Framework of Brans-Dicke Theory
}

\author{
Sudipto Roy \\ Department of Physics, St. Xavier's College, Kolkata, 30 Mother Teresa Sarani (Park Street), \\ Kolkata - 700016, West Bengal, India.
}

\begin{abstract}
On the basis of an assumption of inter-conversion of matter and dark energy, a theoretical model of cosmic expansion has been formulated, in the framework of Brans-Dicke (BD) theory. Empirical expressions of scale factor $(a)$ and scalar field parameter $(\varphi)$ have been used. The scale factor is such that it generates a signature flip of the deceleration parameter with time. A function of time $f(t)$, defined in terms of the density of matter $(\rho)$, is used to account for the non-conservation of matter. This function is proportional to the matter content of the universe. Using an empirical scalar field parameter, in terms of scale factor, the functional form of $f(t)$ has been determined from the BD field equations. The present study reveals that $f(t)$ decreases with time, indicating a conversion of matter into dark energy. Based on this function, the time variation of the density of matter $(\rho)$ has been determined. Expressions regarding the proportions of matter and dark energy of the universe have been derived. The time dependence of gravitational constant $(G), \dot{G} / G$ and the $B D$ dimensionless parameter $\omega(\varphi)$ have been explored. The functional dependence of BD parameter $\omega(\varphi)$ upon the scalar field $(\varphi)$ has been formulated. The study based on the present model establishes a correlation between the transformation of matter content into dark energy and the change of cosmological parameters connected to cosmic expansion, without employing any particular model of interaction between scalar field and matter.
\end{abstract}

Keywords: Cosmology, Brans-Dicke parameter, Scalar field, Conversion of matter into dark energy, Time varying gravitational constant, Accelerated expansion of the universe.

\section{Introduction}

Through a number of recent cosmological observations it has been confirmed that the universe is expanding with acceleration [1-3]. Several studies on cosmology have revealed that nearly seventy percent of all constituents of the universe have a role on its expansion which is opposite to the effect of gravitational attraction and it is referred to as dark energy (DE), which is regarded as responsible for the accelerated expansion of the universe. Its true nature has not yet been determined. The cosmological constant $(\lambda)$ is a widely known parameter of the general theory of relativity, which is referred to as one of the most suitable candidates acting as the source for this repulsive gravitational effect and it agrees with the observational data reasonably well, despite its own shortcomings [4]. So far the researchers have proposed a large number of models regarding dark energy and their characteristics have been studied extensively [4, 5]. It is important to note that this accelerated expansion is a very recent phenomenon and it follows a phase of expansion of universe with deceleration. For the successful nucleosynthesis and also for the structure formation of the universe, this is important. On the basis of observational findings, beyond a certain value of the redshift $(z)($ i.e. $z>1.5)$, the universe certainly had a decelerated phase of expansion [6]. Hence, the evolution of the dark energy component has been such that its effect on the dynamics of the universe is appreciable only during the later stages of the matter dominated era. A recent study by Padmanabhan and Roy Choudhury, based on an analysis of supernova data, has shown that there has definitely been a signature flip of the deceleration parameter $(q)$ of the universe, from positive to negative, indicating a change of phase from deceleration to acceleration [7].

In addition to the models where the effect of dark energy is represented by the cosmological constant $(\lambda)$, many other models regarding the accelerated expansion of universe have been proposed and applied for the sake of a proper explanation of findings [8,9]. Each of these models has successfully generated by a negative value of the deceleration parameter, implying accelerated expansion. One of the most significant of these models is a scalar field with a positive potential which produces an effective negative pressure if the kinetic term is dominated by the potential term. One refers to this scalar field as the quintessence scalar field. In scientific literature there are a large number of models regarding quintessence potentials and they have been extensively used. To have detailed information in this regard one may go through a study by V Sahni on this field [10]. Regarding the origins of models of most of the quintessence potentials, there has been no proper physical explanation.

To explain the findings by using all these different models, one often needs to take into consideration the possibility of an interaction between matter and dark energy, causing probably a transfer of energy from one form to another. Many theories have been proposed where a transfer of energy takes place from dark matter to 
dark energy, in such a manner that over the later period of evolution, the dark energy predominates over matter, causing an accelerated expansion of universe [11, 12]. But the interactions between two components, upon which the models were constructed, were chosen to be arbitrary in most cases, without a strong logical foundation of a physical theory. To determine the role of dark energy in causing accelerated expansion, people have been looking for a theory of interaction between matter and scalar field, using which a cosmologically viable model can be formulated.

To find a way out the discrepancies of these models in the formulation of a particular quintessence field, non-minimally coupled scalar field theories have been used to account for the transition from the decelerated phase to the accelerated phase of cosmic expansion. The presence of the scalar field in the framework of the theory has made it possible and it need not be incorporated separately. The Brans-Dicke (BD) theory, which is regarded as the scalar-tensor generalization of general relativity (GR), is the most natural choice in this context because of its simplicity and a possible reduction to GR in some limit. Thus the BD theory or its modified versions have been shown to account for the present acceleration of universe [13, 14]. An observation regarding the BD theory is that it can potentially generate sufficient acceleration in the matter dominated era even without taking into consideration an exotic quintessence field [15]. However, the researchers have been looking for a theory which can explain the change of cosmic expansion state from deceleration to acceleration. In most of the models the dark energy and dark matter components are considered to be non-interacting and are allowed to evolve independently. Due to the unknown nature of these two components, one is expected to get a relatively generalized framework for study by assuming an interaction between them. It has been shown by Zimdahl and Pavon that the interaction between dark energy and dark matter can be used effectively to solve the coincidence problem [16]. Using this concept one may formulate an interaction of between the dark matter and the BD scalar field. A prediction made by Amendola says that there is a possibility of an inter-conversion of energy between the matter content of the universe and the nonminimally coupled scalar field [17].

Several studies based on BD theory show that, the dimensionless parameter $\omega$ is required to have a very low value, of the order of unity, for an accelerated expansion of the universe [18]. Once it was shown that, assuming the BD scalar field to be interacting with the dark matter, a generalized form of the BD theory can account for an accelerated expansion even with a high value of $\omega$ [19]. For these studies, either one makes a modification of the BD theory to account for the findings properly or a quintessence scalar field is chosen to cause the required acceleration. In a recent study by Clifton and Barrow, and also by Banerjee and Das, it was found that no additional potential is necessary to cause a change of sign of the deceleration parameter from positive to negative $[19,20]$. They considered an interaction between the BD scalar field and the dark matter to explain the observational findings.

A generalized form of BD theory has been used in the present study. Bergman proposed this and Nordtvedt expressed it in a more useful form [21, 22]. In this form, one regards the BD dimensionless parameter $\omega$ as a function of the $\mathrm{BD}$ scalar field $\varphi$, instead of treating it as a constant. No specific mechanism of conversion of matter into dark energy has been used here. The present model is based on an assumption that matter is being converted into dark energy, which is regarded as the sole agent causing the accelerated expansion of the universe. In order to account for the non-conservation of matter, a new function $f(t)$ has been defined as $f(t)=\rho a^{3} / \rho_{0} a_{0}^{3}$. It is clear from this relation that if we consider $f(t)=1$ at all time, the expression of $\rho$ represents conservation of the matter content of the universe. In BD field equations, we have used an empirical expression of scale factor $(a)$ and scalar field parameter $(\varphi)$ to obtain the expressions of $f(t)$. According to its definition, $f(t)$ is proportional to the matter content of the universe at any time $t$. We have explored the behaviour this function to get an estimate of the time evolution of matter and dark energy of the universe, assuming the dark energy to have been produced entirely from matter. The time variation of matter density and the gravitational constant $(G)$ and also the time dependence of the proportions of matter and dark energy of the universe have been explored. This study has found a link between the depletion of the matter content and the change of deceleration parameter and gravitational constant. The rate at which dark energy is produced from matter seems to have a clear influence upon the rates of change of gravitational constant and the deceleration parameter.

\section{Field Equations And The Theoretical Model}

The action in the generalized Brans-Dicke theory is expressed as [23],

$S=\int\left[\frac{\varphi R}{16 \pi G}+\frac{\omega(\varphi)}{\varphi} \varphi_{, \mu} \varphi^{\mu}+L_{m}\right] \sqrt{-g} d^{4} x$

where $R$ is the Ricci scalar, $L_{m}$ is the matter Lagrangian, $\varphi$ is the Brans-Dicke scalar field and $\omega$ is a dimensionless parameter which is considered to be a function of $\varphi$ here instead of being regarded as a constant. For a spatially flat Friedmann-Robertson-Walker space-time, the line element is given by, 
$d s^{2}=d t^{2}-a^{2}(t)\left[d r^{2}+r^{2} d \theta^{2}+r^{2} \sin ^{2} \theta d \phi^{2}\right]$

Here $a$ is the scale factor of the Universe. For a zero curvature Friedmann-Robertson-Walker space-time, variation of action (1) with respect to the metric tensor components yields the following field equations of the generalized Brans-Dicke theory [24].

$3 H^{2}=\frac{\rho}{\varphi}+\frac{\omega(\varphi)}{2}\left(\frac{\dot{\varphi}}{\varphi}\right)^{2}-3 H \frac{\dot{\varphi}}{\varphi}$

$2 \dot{H}+3 H^{2}=-\frac{\omega(\varphi)}{2}\left(\frac{\dot{\varphi}}{\varphi}\right)^{2}-2 H \frac{\dot{\varphi}}{\varphi}-\frac{\ddot{\varphi}}{\varphi}$

Here $H=\frac{a}{a}$ is the Hubble parameter in the above equations. Combining the equations (3) and (4) one gets,

$2 \dot{H}+6 H^{2}=\frac{\rho}{\varphi}-5 H \frac{\dot{\varphi}}{\varphi}-\frac{\ddot{\varphi}}{\varphi}$

From equation (3), the expression of $\omega(\varphi)$ is obtained as,

$\omega(\varphi)=2\left[3 H^{2}-\frac{\rho}{\varphi}+3 H \frac{\dot{\varphi}}{\varphi}\right]\left(\frac{\dot{\varphi}}{\varphi}\right)^{-2}$

In several theoretical studies, where the content of matter of the universe, both dark and baryonic, is assumed to be conserved, one writes the following expression regarding the density of matter [24].

$\rho a^{3}=\rho_{0} a_{0}^{3}=\rho_{0} \quad\left(\right.$ taking $\left.a_{0}=1\right)$

In the framework of Brans-Dicke theory, one comes across studies where one takes into account an interaction between matter and scalar field and it leads to a possibility of inter-conversion between matter and dark energy. To discuss the behaviour of cosmic expansion under this regime of inter-conversion, we define a function $f(t)$ in the following way.

$f(t)=\frac{\rho a^{3}}{\rho_{0} a_{0}^{3}}=\frac{\rho a^{3}}{\rho_{0}} \quad$ (taking $\left.a_{0}=1\right)$

No specific mechanism of interaction between matter and the scalar field has been assumed or used in the present study. The present formulation is based upon an assumption of non-conservation of matter and therefore equation (7) is no longer valid. The dark energy, which is responsible for the accelerated expansion of the universe is assumed to have been generated at the cost of matter. We have attempted to describe these facts quantitatively in terms of the function $f(t)$, defined by equation (8). Thus, $f(t)$ can be regarded as proportional to the total content of matter (dark+baryonic) $M(t)=\rho a^{3}$ of the universe at the instant of time $t$.

Let us denote the ratio of $M(t)$ to $M\left(t_{0}\right)$ by the symbol $R_{1}$ where $R_{1} \equiv f(t)=M(t) / M\left(t_{0}\right)$. Another such ratio $R_{2}=\frac{1}{f} \frac{d f}{d t}=\frac{1}{M} \frac{d M}{d t}$ represents the fractional change of matter per unit time. Negative value of $R_{2}$ indicates a loss of matter, probably due to its transformation into some other entity, may be dark energy, on account of its interaction with the scalar field. Using the function $f(t)$ one may also define a ratio $R_{3}=f-1=\frac{M(t)-M\left(t_{0}\right)}{M\left(t_{0}\right)}$ indicating a fractional change of matter content of the universe with respect to its present value.

The process of conversion of matter into dark energy may be assumed to have started in the past at the time of $t=\gamma t_{0}$ where $\gamma<1$. Hence, the matter content of the universe at $t=\gamma t_{0}$, i.e., $M\left(\gamma t_{0}\right)=M\left(t_{0}\right) R_{1}\left(\gamma t_{0}\right)$ can be regarded as the total content of matter and dark energy at all time. Assuming matter to be the only source of dark energy, the proportion of dark energy in the universe at any time $t$ is given by the following ratio $\left(R_{4}\right)$.

$\boldsymbol{R}_{4}=\frac{M\left(\gamma t_{0}\right)-M(t)}{M\left(\gamma t_{0}\right)}=\frac{f\left(\gamma t_{0}\right)-f(t)}{f\left(\gamma t_{0}\right)}=\frac{\rho\left(\gamma t_{0}\right) a\left(\gamma t_{0}\right)^{3}-\rho(t) a(t)^{3}}{\rho\left(\gamma t_{0}\right) a\left(\gamma t_{0}\right)^{3}}$

Hence, the percentage of dark energy present in the universe would be $\left(\boldsymbol{R}_{\mathbf{4}} \times \mathbf{1 0 0}\right)$. Approximately seventy percent of the total matter-energy content of the universe is dark energy at the present time [18]. For a proper choice of $\gamma$ and another parameter $\boldsymbol{k}$ (to be defined later), we must have $\boldsymbol{R}_{\mathbf{4}}\left(\boldsymbol{t}_{\mathbf{0}}\right) \times \mathbf{1 0 0}=\mathbf{7 0}$ approximately. 
The proportion of matter of the universe, both dark and baryonic, is expressed as,

$\boldsymbol{R}_{\mathbf{5}}=\mathbf{1}-\boldsymbol{R}_{\mathbf{4}}=\mathbf{1}-\frac{M\left(\gamma t_{0}\right)-M(t)}{M\left(\gamma t_{0}\right)}=\frac{M(t)}{M\left(\gamma t_{0}\right)}=\frac{f(t)}{f\left(\gamma t_{0}\right)}=\frac{\rho(t) a(t)^{3}}{\rho\left(\gamma t_{0}\right) a\left(\gamma t_{0}\right)^{3}}$

Hence, the percentage of matter, among the constituents of the universe is $\left(\boldsymbol{R}_{\mathbf{5}} \times \mathbf{1 0 0}\right)$.

The main objective of the present study is to determine a functional form of $f(t)$ to determine the time dependence of the ratios $R_{1}, R_{2}, R_{3}, R_{4}$ and $R_{5}$.

Using these parameters, the density of dark energy can be expressed as,

$\rho_{d}=\left(R_{4} / R_{5}\right) \rho=\frac{f\left(\gamma t_{0}\right)-f(t)}{f(t)} \rho=\frac{\rho\left(\gamma t_{0}\right) a\left(\gamma t_{0}\right)^{3}-\rho(t) a(t)^{3}}{\rho(t) a(t)^{3}} \rho$

On the basis of equation (8), the expression of $f(t)$ can be written as,

$f(t)=a^{3} \frac{\rho}{\rho_{0}}$

In the above expression, the density of matter $(\rho)$ can be obtained from equation (5). For this purpose one needs to choose some suitable functional form of the Brans-Dicke scalar field $\varphi$. Following some recent studies in this regard we have chosen an empirical forms of $\varphi,[24,25]$. This empirical expression is,

$\varphi=\varphi_{0}\left(\frac{a}{a_{0}}\right)^{k}=\varphi_{0} a^{k}$

The constant $k$ in equation (13) determines the rapidity with which the parameter $\varphi$ changes with time. According to Brans-Dicke theory, the gravitational constant $=\frac{1}{\varphi}$.

The following expression of the density of matter $(\rho)$ of the universe is obtained by combining equation (13) with equation (5).

$\rho=\varphi H^{2}\left[k^{2}+(4-q) k+(4-2 q)\right]$

Using equation (14), $\rho_{0}$ can be written as,

$\rho_{0}=\varphi_{0} H_{0}{ }^{2}\left[k^{2}+\left(4-q_{0}\right) k+\left(4-2 q_{0}\right)\right]$

Substituting from equations (14) and (15) into equation (12) we get,

$f(t)=a^{3} \frac{\rho}{\rho_{0}}=a^{3} \frac{\varphi H^{2}\left[k^{2}+(4-q) k+(4-2 q)\right]}{\varphi_{0} H_{0}^{2}\left[k^{2}+\left(4-q_{0}\right) k+\left(4-2 q_{0}\right)\right]}$

In deriving the expression of $\rho$ in equation (14), we have used the standard expressions of Hubble parameter $(H)$ and deceleration parameter $(q)$, which are, $H=\dot{a} / a$ and $q=-\ddot{a} a / \dot{a}^{2}$ respectively. In the expression of the function $f(t)$, in equation (16), the parameters $\varphi, H$ and $q$ are all functions of time . Their time variation depends upon the time evolution of the scale factor $(a)$. To determine $f(t)$, using equation (16), an empirical scale factor has been used. This scale factor has been so chosen that the deceleration parameter $q\left(\equiv-\ddot{a} a / \dot{a}^{2}\right)$ calculated from it shows a signature flip as a function of time. This is in accordance with the well known observation that the universe has changed its phase of expansion from deceleration to acceleration $[18,19,24]$. This empirical scale factor is expressed as,

$a=a_{0} \operatorname{Exp}\left[-\alpha t_{0}^{\beta}\right] \operatorname{Exp}\left[\alpha t^{\beta}\right]$

Here $\alpha, \beta$ are constants. Here $\beta>0$ to make sure that the scale factor increases with time. The scalar field parameter $(\varphi)$, Hubble parameter $(H)$ and deceleration parameter $(q)$, based on this scale factor are given by,

$\varphi=\varphi_{0}\left(\frac{a}{a_{0}}\right)^{k}=\varphi_{0} \operatorname{Exp}\left[-k \alpha t_{0}^{\beta}\right] \operatorname{Exp}\left[k \alpha t^{\beta}\right]$

$H=\dot{a} / a=\alpha \beta t^{(\beta-1)}$

$q=-\ddot{a} a / \dot{a}^{2}=-1+\frac{1-\beta}{\alpha \beta} t^{-\beta}$

Here we find that, for $0<\beta<1$ and $\alpha>0$ we have,

$q>0$ for $t=0$ and $q \rightarrow-1$ as $t \rightarrow \infty$

It clearly means that the chosen scale factor generates an expression of deceleration parameters which changes sign from positive to negative as time goes on. The values of constant parameters $(\alpha, \beta)$ have been determined from the following conditions.

Condition 1: $\quad H=H_{0}$ at $t=t_{0}$

Condition 2: $\quad q=q_{0}$ at $t=t_{0}$

Combining the equations (21) and (22) with the equations (19) and (20) respectively, one obtains $\alpha=6.39 \times$ $10^{-10}$ and $\beta=5.37 \times 10^{-01}$

The values of different cosmological parameters used in the present study are, 
$H_{0}=2.33 \times 10^{-18} \mathrm{sec}^{-1}, t_{0}=4.415 \times 10^{17} \mathrm{sec}, \varphi_{0}=\frac{1}{G_{0}}=1.498 \times 10^{10} \mathrm{~m}^{-3} \mathrm{Kg} \mathrm{s}^{2}$
$\rho_{0}=2.831 \times 10^{-27} \mathrm{Kg} / \mathrm{m}^{3}$ and $q_{0}=-0.55$

To determine the value of $f(t)$ from the equations (16), one must use the expressions of (18), (19) and (20) and also the values of the constants $\alpha$ and $\beta$. Thus we get,

$f(t)=\frac{\operatorname{Exp}\left[\alpha(3+k)\left(t^{\beta}-t_{0} \beta\right)\right]\left\{-1+\beta+\alpha \beta(3+k) t^{\beta}\right\}\left(\mathrm{t} / \mathrm{t}_{0}\right)^{\beta-2}}{-1+\beta+\alpha \beta(3+k) \mathrm{t}_{0}{ }^{\beta}}$

The defining expression of $f(t)$ is $\rho a^{3}=f(t) \rho_{0} a_{0}^{3}$. According to this relation, the value of $f(t)$ is always positive and, $f(t)=1$ at $t=t_{0}$ (taking $a_{0}=1$ ). The form of the function $f(t)$ in equation (16) and (23) are such that $f(t)=1$ at $t=t_{0}$. The values of $k$ for which $f(t)$ is positive over the entire range of study (say, from $t=0.5 t_{0}$ to $\left.t=1.5 t_{0}\right)$ is given below.

$k<\left(k_{-}\right)_{\min }$ or $k>\left(k_{+}\right)_{\max }$ over the entire range of study. Here,

$\left(k_{-}\right)_{\min }=(q-2)_{\min } \quad$ over the range from $t=0.5 t_{0}$ to $t=1.5 t_{0}$

$\left(k_{+}\right)_{\max }=(q-2)_{\max } \quad$ over the range from $t=0.5 t_{0}$ to $t=1.5 t_{0}$

For our range of study, i.e. from $t=0.5 t_{0}$ to $t=1.5 t_{0}$, we find, using equation (20),

$\left(k_{-}\right)_{\min }=-2.64$ and $\left(k_{+}\right)_{\max }=-2.33$

Here we have a lower and an upper range of permissible values for $k$ which are $k<\left(k_{-}\right)_{\min }$ or $k>\left(k_{+}\right)_{\max }$ respectively. The upper range, $k>\left(k_{+}\right)_{\max }$, includes both positive and negative values of $k$ and the lower range, $k<\left(k_{-}\right)_{\min }$, has only negative values. As per equation (13), the upper range of $k$ allows $G=\frac{1}{\varphi}$ to be both increasing and decreasing functions of time, although for the lower range of $k$ values $G$ becomes an increasing function of time. To choose the proper range of $k$, we have determined the values of $\omega_{0}$ at different values of $k$ and compared them with those obtained from other studies. Using equation (6) we can write the following expression (eqn. 26) regarding $\omega$ for this model.

$\omega=\frac{2}{k^{2}}\left[3(1+k)-\frac{\rho}{\phi H^{2}}\right]$

Using equations (26) we get the following expression of $\omega_{0}$ (the value of $\omega$ at the present epoch).

$\omega_{0}=\frac{2}{k^{2}}\left[3(1+k)-\frac{\rho_{0}}{\phi_{0} H_{0}^{2}}\right]$

Several studies reveal that $\omega_{0}$ has a negative value close to $(-1)$ [18].

To have $\omega_{0}<0$, the condition to be satisfied by $k$ is given by,

$k<\frac{\rho_{0}}{3 \phi_{0} H_{0}^{2}}-1$ or, $k<-0.9884$

For the whole of the lower range of $k$ values and for a part of its upper range, the above condition is satisfied. The gravitational constant $(G)$, is reciprocal of the Brans-Dicke scalar field parameter $(\varphi)$. Thus, from equation (18) we get,

$\frac{G}{G_{0}}=\frac{\varphi_{0}}{\varphi}=\operatorname{Exp}\left[k \alpha t_{0}^{\beta}\right] \operatorname{Exp}\left[-k \alpha t^{\beta}\right]$

A parameter $\dot{G} / G$, experimentally measured by various means, is obtained from (29) as,

$\frac{\dot{G}}{G}=\frac{1}{G} \frac{d G}{d t}=-k \frac{\dot{a}}{a}=-k H$

Using equation (30) we get,

$\left(\frac{\dot{G}}{G}\right)_{t=t_{0}}=-k H_{0}$

The well accepted upper limit of $\left(\frac{\dot{G}}{G}\right)_{t=t_{0}}$ is $4 \times 10^{-10} \mathrm{Yr}^{-1}[26]$.

\section{Graphical Interpretation Of Theoretical Findings}

A plot of $(\dot{G} / G)_{t=t_{0}}$ as a function of $k$, based on equation (31) is shown in Figure 1. Its positive value indicates that $G$ is presently increasing with time and negative value indicates its reverse behaviour. For the entire lower range of $k$ values it is positive. For the upper range of $k$ values it is found to be both positive and negative. There are experimental observations and theoretical models where $G$ has been shown to be increasing with time [27]. 
We have plotted $\omega_{0}$ as a function of $k$ in Figure 2. For the lower range of $k$ values, the values of $\omega_{0}$ are negative and also close to the values obtained from other studies [18]. For the upper range of $k$, we have both positive and negative values of $\omega_{0}$, the positive values being totally contrary to the findings of other studies in this regard [18]. This observation is in favour of using the lower ranges of $k$ values.

Figure 3 shows the variation of $R_{1}(\equiv f)$ as a function of time for three different values of $k$ in its lower range. It shows that the matter content of the universe $\left[M(t)=f(t) M_{0}\right]$ decreases with time and the rate of its reduction is faster for more negative values of $k$.

It is evident from Figure 4 that the Brans-Dicke parameter $\omega$ is an increasing function of time, with only negative values, for the lower range of $k$ values. But its behavior is different for the upper range of $k$ values. For the positive values of the upper range of $k$, the values of $\omega$ are all positive which is inconsistent with the findings of other studies [18].

On the basis of these findings from the figures 1,2 and 4, we have found it logical to chose values from the lower range of $k$ to determine the time dependence of $f(t)$ and other relevant parameters connected to it in the present study.

Figure 5 shows the plots of $R_{4}$ and $R_{5}$ as functions of time. The proportion of dark energy $\left(R_{4}\right)$ in the universe is found to increase with time. Since it is assumed to be generated at the cost of matter (dark + baryonic), the proportion of matter in the universe $\left(R_{5}\right)$ decreases with time. Thus, the sum of $R_{4}$ and $R_{5}$ is unity since the beginning of the conversion process at $t=\gamma t_{0}$.

The variation of $R_{2}$ and $R_{3}$ as functions of time for two different values of $k$, in its lower range, has been shown in Figure 6. It shows that $R_{2}$, the fractional rate of change of matter content is always negative, implying a transformation from matter to other energy forms. Its value decreases with time, indicating a slowing down of the transformation process as time goes on. As $k$ values become more negative, transformation rate becomes larger. The values of $R_{3}$ are positive in the past $\left(t<t_{0}\right)$ and negative in future $\left(t>t_{0}\right)$, as expected from the time variation of $R_{1}$ and $R_{2}$. It also changes faster for more negative values of $k$.

Figure 7 shows the variation of $q$ and $G / G_{0}$ as functions of $R_{4}$, the proportion of dark energy of the universe. These are based on equations (20) and (29). As $R_{4}$ increases, the deceleration parameter becomes more negative, indicating greater acceleration of the cosmic expansion. The gravitational constant increases as the dark energy proportion of the universe increases. Therefore the dark energy may be assumed to have a role in causing accelerated expansion and also in increasing the gravitational constant.

Two of the three plots of figure 8 show the variation of matter density $(\rho)$ of the universe as a function of time for two different values of $k$ in its lower range. These plots are based on equation (8). These curves show that the density decreases at a faster rate for more negative values of the parameter $k$. The third plot of this figure is based on equation (7) which shows the variation of matter density when the matter is assumed to remain conserved. For that curve we have assumed that $f(t)=1$ for all values of $t$. Plots corresponding to the non-conservation regime shows a faster fall than that for the conservation of matter. For the third plot $(f=1)$, the density of matter changes only due to the expansion of the universe. For two other plots, the density changes due to the expansion of the universe and also the conversion of matter into some other form of energy.

All these plots show that as $k$ is made more and more negative, the rate of reduction of matter content (proportional to $R_{1}$ ) and the rate of change of its density $(\rho)$ increases and the rates of change of gravitational constant and deceleration parameter also increase. It indicates clearly that there exists a relation of the transformation of matter into dark energy with the gravitational constant and state of expansion of the universe characterized by the deceleration parameter and its time variation.

\section{Conclusion}

The purpose of the present study is to determine the nature of variation of several cosmological parameters, which characterize the cosmic expansion, by theoretically incorporating an assumption of nonconservation of the matter content of the universe in the field equations of Brans-Dicke theory. In these field equations, an expression of the density of matter $(\boldsymbol{\rho})$ has been used, which was constructed on an assumption of inter-conversion between matter and dark energy. In this model, the density of matter depends upon a function of time $\boldsymbol{f}(\boldsymbol{t})$, which is proportional to the matter content of the universe at any instant of time. We have not assumed any form of this function while incorporating this function into the expression of density. Its form has been determined from the field equations. To improve this model one may choose a particular form of this function that actually accounts for the non-conservation of matter-energy. The preset study shows that, this function, which is proportional to the matter content of the universe, decreases with time, clearly indicating a particular direction of conversion, which is from the matter component of the universe to its dark energy component. It is found through graphical analysis that, as the proportion of dark energy content of the universe increases with time, the deceleration parameter becomes more and more negative and the gravitational constant is found to increase with time. All these observations are clearly implying a possibility that there is a dependence of the rate of change of gravitational constant and the deceleration parameter on the content of dark 
energy and the rate at which it changes. It has been revealed by the present model that if the dark energy is considered to be produced completely at the cost of matter (both dark and baryonic), its present proportion should depend on the time at which the process of conversion of matter into energy began in the universe. The present study shows that, by a proper tuning of parameters, it is possible to obtain values from the theoretical model, which are close to the experimental evidence regarding the present proportions of dark energy and matter. In order to continue this study in future, we have plans to improve this model by choosing a different empirical form of the scale factor $(\boldsymbol{a})$ and also by using a new form of the Brans-Dicke scalar field parameter $(\boldsymbol{\varphi})$. A new form of the function $\boldsymbol{f}(\boldsymbol{t})$, expressed in terms of scale factor $(\boldsymbol{a})$ and the scalar field parameter $(\boldsymbol{\varphi})$, can also be chosen for this purpose. One must make sure that $\boldsymbol{f}(\boldsymbol{t})$ is unity at the present time $\left(\boldsymbol{t}=\boldsymbol{t}_{\mathbf{0}}\right)$, according to its defining relation of equation (8). The time dependence of several such empirical forms of this function can be studied to determine the time evolution of the contents of matter and dark energy of the universe, the rate of their inter-conversion and their influence upon the time evolution of various factors governing the accelerated expansion of the universe.

Figures

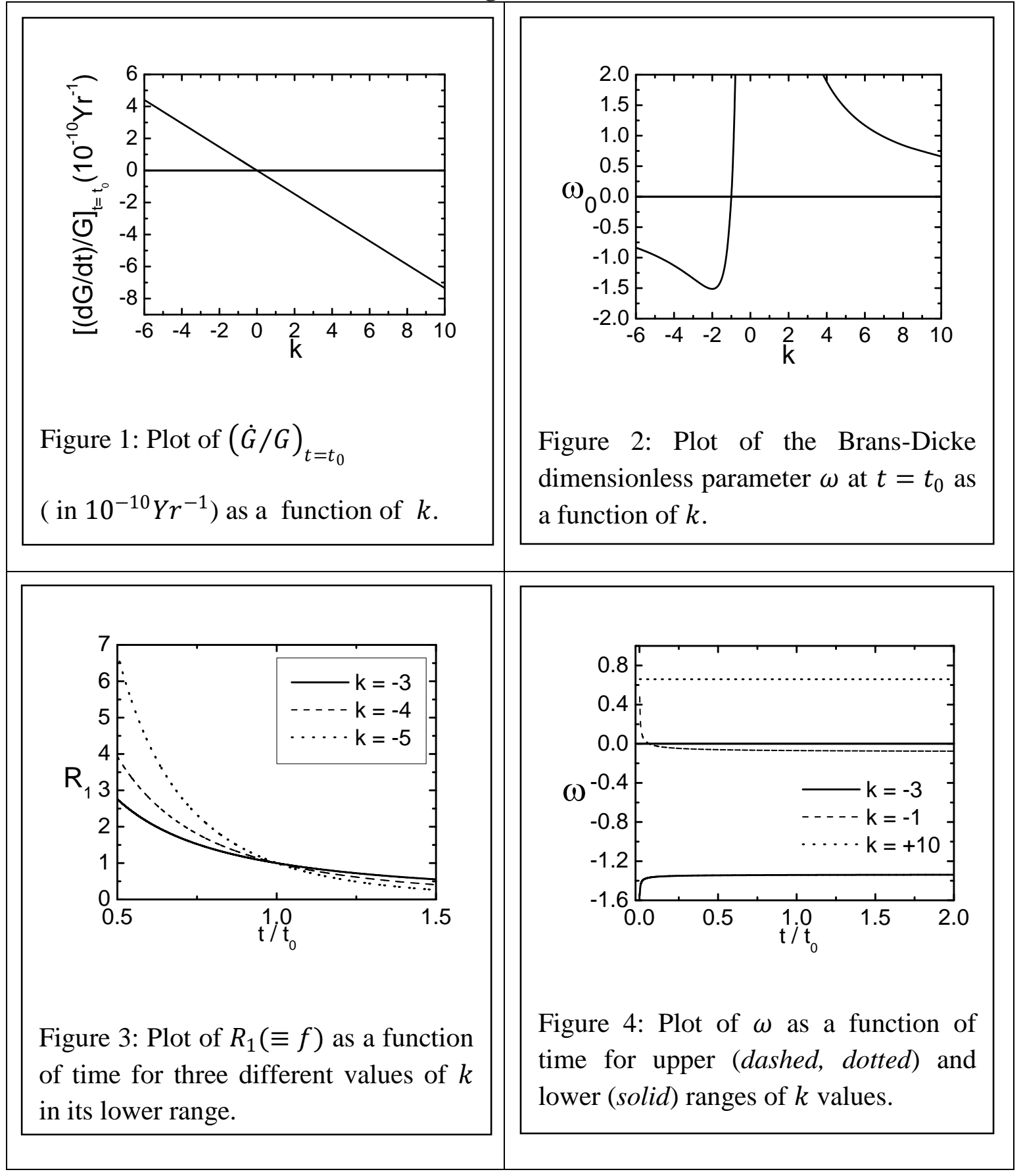




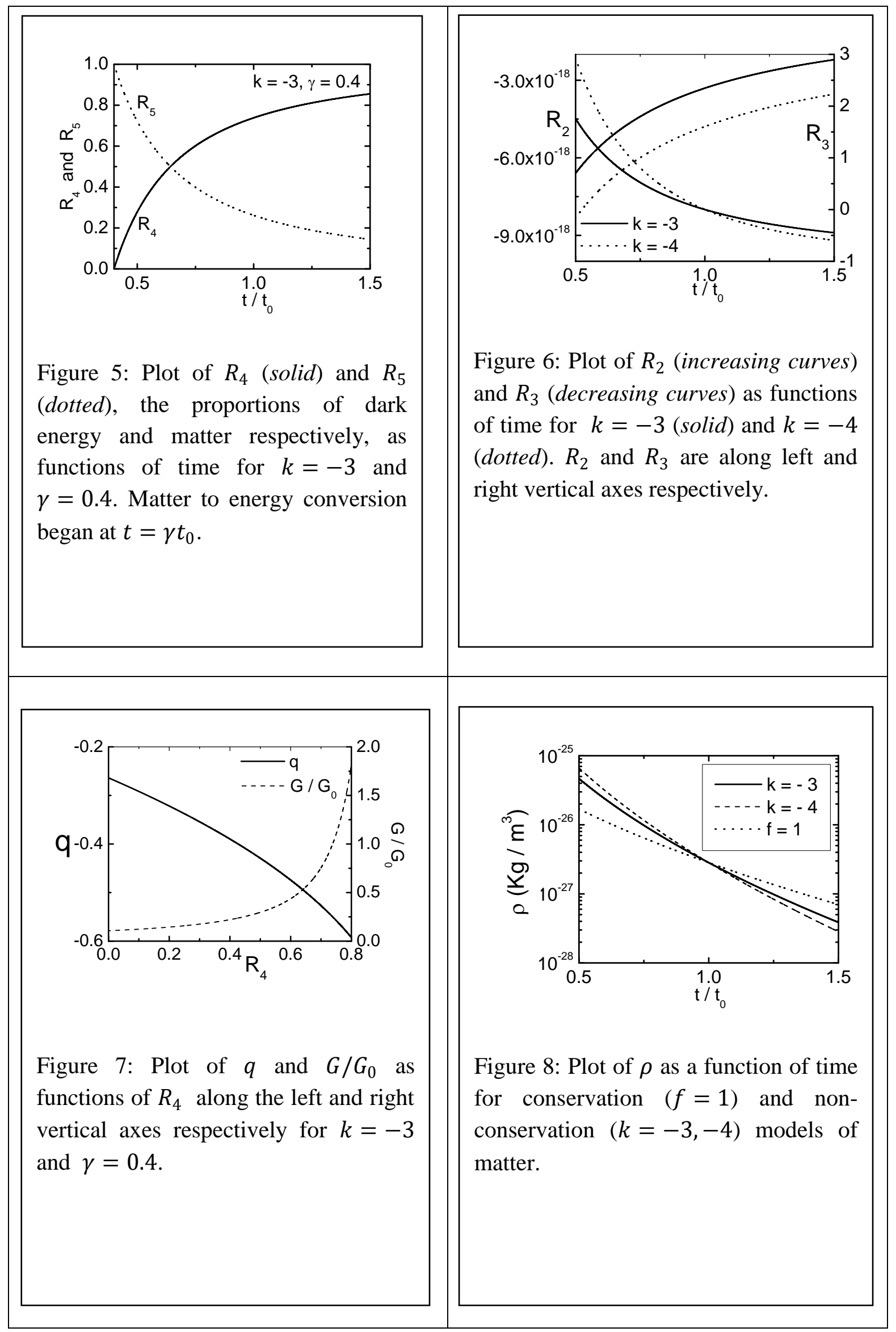




\section{References}

[1]. Bennet C. L. et al.; 2003. First-Year Wilkinson Microwave Anisotropy Probe (WMAP) Observations: Preliminary maps and basic results. The Astrophysical Journal Supplement Series, 148(1): 1-27.

[2]. Riess A. G. et al.; 1998. Observational Evidence from Supernovae for an accelerating universe and a cosmological constant. Astronom. J., 116(3): 1009-1038.

[3]. Perlmutter S. et al.; 1999. Measurements of Omega and Lambda from 42 High-Redshift Supernovae. Astrophys. J., 517: $565-586$.

[4]. Sahni V. and Starobinsky A.; 2000. The case for a positive cosmological $\Lambda$ term. Int. J. Mod. Phys. D, 09(4): $373-443$.

[5]. Padmanabhan T.; 2003.. Cosmological Constant-the weight of the vacuum. Phys. Rept., 380: 235-320.

[6]. Riess A. G. et al.; 2001. The farthest known supernova: support for an accelerating universe and a glimpse of the epoch of deceleration. Astrophys. J., 560: 49-71.

[7]. Padmanabhan T. and Roy Choudhury T.; 2003. A theoretician's analysis of the supernova data and the limitations in determining the nature of dark energy. Mon. Not. R. Astron. Soc., 344: 823-834.

[8]. Copeland E. J., Sami M. and Tsujikawa S.; 2006. Dynamics of dark energy. Int. J. Mod. Phys. D, 15: 1753-1936.

[9]. Martin J.; 2008. Quintessence: a mini-review. Mod. Phys. Lett. A, 23: 1252-1265.

[10]. Sahni V.; 2004. Dark Matter and Dark Energy. Lect. Notes Phys., 653: 141-180.

[11]. Zimdahl W.; 2012. Models of Interacting Dark Energy. arXiv:1204.5892v1 1-6.

[12]. Reddy D. R. K., Kumar R. S.; 2013. Two Fluid Scenario for Dark Energy Model in a Scalar-Tensor Theory of Gravitation. Int. J. Theor. Phys., 52: 1362-1369.

[13]. Banerjee N. and Pavon D.; 2001a. Cosmic acceleration without quintessence. Phys. Rev. D., 63: 043504-043508.

[14]. Brunier T., Onemli V. K. and Woodard R. P.; 2005. Two loop scalar self-mass during inflation. Class. Quantum Grav., 22: 59-84.

[15]. Banerjee N. and Pavon D.; 2001b. A quintessence scalar field in Brans-Dicke theory. Class. Quantum Grav., 18: 593-599.

[16]. Zimdahl W., Pavon D.; 2004. Interacting quintessence and the coincidence problem. arXiv:astro-ph/0404122v1 1-3.

[17]. Amendola L.; 1999. Coupled Quintessence. Phys. Rev. D., 62: 043511-043520.

[18]. Das S. and Mamon A. A.; 2014. An Interacting model of Dark Energy in Brans-Dicke theory. Astrophys. Space Sci., 351: $651-660$.

[19]. Banerjee N. and Das S.; 2006. An interacting scalar field and the recent cosmic acceleration. Gen. Relativ. Gravit., 38 (5): $785-794$.

[20]. Clifton T. and Barrow J. D.; 2006. Decaying Gravity. arXiv:gr-qc/0603116v2 1-13.

[21]. Bergman P. G.; 1968. Comments on the scalar-tensor theory. Int. J. Theor. Phys., 1(1): 25-36.

[22]. Nordtvedt K.; 1970. Post-Newtonian metric for a general class of scalar-tensor gravitational theories and observational consequences. Astrophys. J., 161: 1059-1067.

[23]. Brans C. and Dicke R. H.; 1961. Mach's Principle and a Relativistic Theory of Gravitation. Phys. Rev., 124: $925-935$.

[24]. Banerjee N. and Ganguly K.; 2009. Generalised Scalar-Tensor theory and the cosmic acceleration. Int. J. Mod. Phys. D, 18 (3): 445-451.

[25]. Roy S., Chattopadhyay S. and Pasqua A.; 2013. A study on the dependence of the dimensionless Brans-Dicke parameter on the scalar field and their time dependence. Eur. Phys. J. Plus, 128 (147): 1-16.

[26]. Weinberg S.; 1972. Gravitation and Cosmology: Principles and Applications of the General Theory of Relativity. John Wiley \& Sons (Asia) Pte. Ltd., Singapore: 630.

[27]. Pradhan A., Saha B. and Rikhvitsky V.; 2015. Bianchi type-I transit cosmological models with time dependent gravitational and cosmological constants: re-examined. Indian J. Phys., 89 (5): 503-513. 